Hitotsubashi University

Graduate School of Economics

Discussion Paper No. 2014-06

\title{
Comparing Preference Orders: Asymptotic Independence
}

Kazuya Kikuchi

May 2014 


\title{
Comparing Preference Orders: Asymptotic Independence*
}

\author{
Kazuya Kikuchi ${ }^{\dagger}$
}

May 2014

\begin{abstract}
A decision maker is presented with two preference orders over $n$ objects and chooses the one which is "closer" to his own preference order. We consider several plausible comparison rules that the decision maker might employ. We show that when $n$ is large and the pair of orders to be compared randomly realizes, different comparison rules lead to statistically almost independent choices. Thus, two people with a common preference relation may nonetheless exhibit almost uncorrelated choice patterns.
\end{abstract}

JEL Code: D01

Keywords: preference relation, rank correlation

*I would like to thank Professors Koichi Tadenuma, Nozomu Muto, and seminar participants at Waseda University for their helpful comments and suggestions.

${ }^{\dagger}$ Graduate School of Economics, Hitotsubashi University, Naka 2-1, Kunitachi, Tokyo 186-8601, Japan. Email: kazuya.kikuchi68@gmail.com 


\section{Introduction}

Consider a questionnaire that asks a voter: "Which candidate, $A$ or $B$, has a policy preference closer to yours?" Unless the meaning of "closer" is provided in advance, even voters with the same policy preference may respond differently. Yet, if the above question is repeated for a sufficiently large variety of candidates and two voters exhibit significantly distinct choice patterns, one might be tempted to conclude that they have distinct policy preferences.

This short paper shows a case where in a general situation like the above, almost uncorrelated choice patterns arise from individuals with a common underlying preference. We consider five comparison rules that map the decision maker's underlying preference over objects (e.g., the voter's preference over policies) to his ranking over preference orders (e.g., his ranking over candidates). One is the Kemeny rule which treats all pairs of objects equally, ${ }^{1}$ and the other four are lexicographic rules which are procedurally simpler. We show that while these rules frequently induce identical comparisons when the number $n$ of objects is small, this similarity disappears as $n$ becomes large. More precisely, when the pair of preference orders to be compared randomly realizes, for large $n$, the comparisons made under the five rules are almost statistically (pairwise) independent; in other words, the Kendall rank correlation coefficients between them converge to 0 .

One way to interpret this result is to assume that the decision maker has some "welfare" preference, as distinguished from his "behavioral" preference, both defined on the set of preference orders (see Rubinstein and Salant (2012)). For instance, suppose a voter must cast a ballot for either candidate $A$ or $B$. Suppose further that after the election, the winning candidate will face the choice between any two policies equally likely. Hence the voter's welfare preference over candidates would be the one which is defined by the Kemeny rule applied to his policy preference. However, the actual choices made by the voter may be inconsistent with this preference. For exam-

${ }^{1}$ This rule is based on the Kemeny distance function (Kemeny and Snell, 1962) defined on the set of linear orders over $n$ objects (the original definition allows for weak orders). The Kemeny distance between two orders is defined to be the number of pairs of objects on which the two orders disagree. See Kemeny and Snell (1962) for an axiomatic characterization of this distance function, and Burak and Storcken (2013) for an improved characterization result. 
ple, he may adopt the quicker decision procedure in which he first compares the top policies for the two candidates according to his own policy preference. If he prefers $A$ 's top policy then he votes for $A$; if he is indifferent, then he compares the second-ranked policies for the candidates, and so on. This procedure induces choices that are consistent with a lexicographic rule. Our result suggests that when the number of policies is large, the distortion in voting behavior caused by employing the alternative procedure is quite large: the behavioral preference coincides with the welfare preference only with probability around one-half.

Some of the comparison rules considered in this paper have already appeared in previous studies, especially in the literature on the incentive problem arising in preference aggregation. ${ }^{2}$ This literature addresses the question of whether and how society can prevent individuals from manipulating the social preference. To describe this problem, one needs to make an assumption about how each individual ranks possible social preference orders based on his own preference order. Bossert and Storcken (1992) assume that individuals follow the Kemeny rule. Sato (2013c) allows for a wide class of comparison rules which include all rules considered here. Bossert and Sprumont (2014) assume a “domination"-type rule. In the case of a voter's comparison between candidates $A$ and $B$, we say that $A$ dominates $B$ (in terms of the voter's policy preference) if the following holds:

D: whenever the voter agrees with candidate $B$ on a pair of policies, he also agrees with candidate $A$ on that pair of policies.

The rule defined by the domination relation is incomplete in that some pairs of preference orders are incomparable. The five comparison rules discussed in this paper are complete and satisfies the domination principle; hence they are different complete extensions of the domination rule.

\footnotetext{
${ }^{2}$ In a somewhat different context, Laffond and Lainé (2000) provide a characterization of two lexicographic rules.
} 


\section{Comparison rules}

\subsection{A voting example}

Suppose there are three political goals,

\{Defense, Equality, Growth\}.

A voter has the priority order

Equality $>$ Growth $>$ Defense.

Two candidates, $A$ and $B$, have the priority orders

$$
\begin{aligned}
& \text { Defense }>_{A} \text { Equality }>_{A} \text { Growth } \\
& \text { Growth }>_{B} \text { Defense }>_{B} \text { Equality. }
\end{aligned}
$$

Which candidate does the voter choose? We discuss five comparison rules that the voter might employ. One is the Kemeny rule which puts equal weights on all possible pairs of goals. The other four are lexicographic rules which are procedurally less demanding.

Kemeny rule. The voter measures his Kemeny distance from each candidate (i.e., the number of pairs of goals on which he disagrees with the candidate) and chooses the candidate closer to him. His distance from each candidate is 2 , since he disagrees with $A$ on \{Defense, Equality $\}$ and \{Defense, Growth\}, and disagrees with candidate $B$ on $\{$ Equality, Growth $\}$ and $\{$ Defense, Equality . Thus in this case, the rule does not select a single candidate.

Descending rule. ${ }^{3}$ The voter first compares the top-priority goals for candidates $A$ and $B$ (i.e., Defense and Growth) according to his own priority order. If he ranks one candidate's top goal

\footnotetext{
${ }^{3}$ See Sato (2013c) for the concept of non-manipulability of social preferences when agents follow the descending rule.
} 
higher then he chooses that candidate; if he is indifferent then he goes on to compare the secondpriority goals for the two candidates, and so on. As Growth $>$ Defense, the voter chooses candidate $B$.

Ascending rule. The voter first compares the bottom-priority goals for candidates $A$ and $B$ (i.e., Equality and Growth). If he ranks one candidate's bottom goal lower then he votes for that candidate; if he is indifferent then he goes on to compare the second-priority goals for the two candidates, and so on. As Equality $>$ Growth, the voter chooses candidate $A$.

Inverse descending rule. The voter first compares the ranks which his top-priority goal (Equality) receives from the two candidates. If one candidate ranks it higher then he chooses that candidate; if the two candidates rank it equally then he goes on to compare the ranks which his secondpriority goal receives, and so on. As candidate $A$ ranks Equality higher than $B$ does, the voter chooses $A$.

Inverse Ascending rule. The voter first compares the ranks which his bottom-priority goal (Defense) receives from the two candidates. If one candidate ranks it lower then he chooses that candidate; if the two candidates rank it equally then he goes on to compare the ranks which his second-priority goal receives, and so on. As candidate $B$ ranks Defense lower than $A$ does, the voter chooses $B$.

\subsection{General definitions}

Formally, let $\{1,2, \ldots, n\}$ be the set of objects. In this paper a (preference or priority) order refers to a linear order over objects. We fix the decision maker's preference order over objects as

$$
1>2>\ldots>n \text {. }
$$


Any order is expressed as a permutation of $\{1, \ldots, n\}$,

$$
\pi=(\pi(1), \ldots, \pi(n)) .
$$

This notation means that " $\pi(i)$ is the $i$ th-ranked object in the order $\pi$." Let $\Pi$ be the set of orders. In principle, a comparison rule is a rule that maps the decision maker's underlying order $>$ to his ordering over orders. However, since we have fixed $>$ as above, we can identify a comparison rule as an ordering on $\Pi$.

An inversion in an order $\pi$ is a pair $\{i, j\}$ of ranks such that $i<j$ and $\pi(i)>\pi(j)$. Denote by $I(\pi)$ the number of inversions in $\pi . I(\pi)$ measures the frequency with which the decision maker disagrees with another person with preference $\pi$.

Our five comparison rules are defined as follows:

- Kemeny rule $\gtrsim_{K}: \pi \gtrsim_{K} \pi^{\prime}$ if and only if $I(\pi) \leq I\left(\pi^{\prime}\right)$.

- Descending rule $\gtrsim_{D}: \pi>_{D} \pi^{\prime}$ if and only if there exists $i \leq n$ such that $\pi(j)=\pi^{\prime}(j)$ for all $j<i$ and $\pi(i)<\pi^{\prime}(i)$ (and $\pi \sim_{D} \pi^{\prime}$ if and only if $\pi=\pi^{\prime}$ ).

- Ascending rule $\gtrsim_{A}: \pi>_{A} \pi^{\prime}$ if and only if there exists $i \leq n$ such that $\pi(j)=\pi^{\prime}(j)$ for all $j>i$ and $\pi(i)>\pi^{\prime}(i)$.

- Inverse descending rule $\gtrsim_{D}^{-}: \pi \gtrsim_{D}^{-} \pi^{\prime}$ if and only if $\pi^{-1} \succsim_{D}\left(\pi^{\prime}\right)^{-1} \cdot{ }^{4}$

- Inverse ascending rule $\gtrsim_{A}^{-}: \pi \succsim_{A}^{-} \pi^{\prime}$ if and only if $\pi^{-1} \succsim_{A}\left(\pi^{\prime}\right)^{-1}$.

The Kemeny rule is at least theoretically appealing especially when the decision maker has no reason to attach more importance to an object pair than another object pair. Even in this case, however, it is not quite obvious that when presented with the order $\pi=2743156$, he approaches it by counting the number of inversions. If alternatively he first pays attention to the object occupying

\footnotetext{
${ }^{4} \pi^{-1}$ denotes the inverse of permutation $\pi$.
} 
the first rank in $\pi$, then it seems plausible that he adopts the descending rule. Likewise, if he first focuses on object 1 , then it seems plausible that he follows the inverse descending rule. On the other hand, if $\pi$ is presented not as a permutation but as a directed graph (with an arc emanating from object $i$ to object $j$ if and only if $\pi(i)<\pi(j))$, then the decision maker might more easily come up with the Kemeny rule than before.

\section{Asymptotic independence}

We consider an experiment in which the decision maker faces a randomly drawn pair of orders $\left(\pi, \pi^{\prime}\right) \in \Pi \times \Pi$. The sample space is $\Pi \times \Pi$ and the probability measure $\mathcal{P}$ assigns $1 / n !^{2}$ to each sample point $\left(\pi, \pi^{\prime}\right)$. Let $\gtrsim^{*}$ and $\gtrsim^{* *}$ be two comparison rules. We say that the two comparison rules are asymptotically independent if

$$
\lim _{n \rightarrow \infty} \mathcal{P}\left\{\pi \gtrsim^{*} \pi^{\prime}, \pi \gtrsim^{* *} \pi^{\prime}\right\}=\lim _{n \rightarrow \infty} \mathcal{P}\left\{\pi \gtrsim^{*} \pi^{\prime}\right\} \mathcal{P}\left\{\pi \gtrsim^{* *} \pi^{\prime}\right\}
$$

Any two of our five rules, $z^{*}$ and $z^{* *}$, are asymptotically independent if $\mathcal{P}\left\{\pi>^{*} \pi^{\prime}, \pi^{\prime}>^{* *} \pi\right\} \rightarrow$ $1 / 4 .^{5}$

Proposition 1. The comparison rules, $\gtrsim_{K}, \gtrsim_{D}, \succsim_{A}, \gtrsim_{D}^{-}$, $\gtrsim_{A}^{-}$, are asymptotically pairwise independent.

We divide the proof into Claim 1 for asymptotic independence between lexicographic rules and Claim 2 for asymptotic independence between the Kemeny rule and lexicographic rules.

Claim 1. The lexicographic comparison rules, $\gtrsim_{D}, \succsim_{A}, \gtrsim_{D}^{-}$, $\gtrsim_{A}^{-}$, are asymptotically pairwise independent.

${ }^{5}$ This is because for any comparison rule $\gtrsim$ considered here, indifference occurs with probability converging to 0 as $n \rightarrow \infty$. This is obvious for lexicographic rules, since they are strict orders on $\Pi$ and hence indifference occurs only if $\pi=\pi^{\prime}$. The Kemeny rule is a weak order. But it is known that when $\pi$ is drawn randomly, a certain normalization of $I(\pi)$ converges in law to a normal distribution (see e.g., Chung (2001)). Hence, for independently drawn $\pi$ and $\pi^{\prime}$, the probability that $\pi \sim_{K} \pi^{\prime}$ (i.e., the probability that $I(\pi)=I\left(\pi^{\prime}\right)$ ) converges to 0 . 
Proof. We only show the asymptotic independence between $\gtrsim_{D}$ and $\gtrsim_{D}^{-}$, and between $\gtrsim_{D}$ and $\gtrsim_{A}$. The remaining part can be similarly shown.

Part 1: $\gtrsim_{D}$ and $\succsim_{D}^{-}$are asymptotically independent.

Proof. We count the number $N$ of pairs $\left(\pi, \pi^{\prime}\right)$ such that $\pi>_{D} \pi^{\prime}$ but $\pi^{\prime}>_{D}^{-} \pi$. This occurs if and only if either: (a) $\pi(1)<\pi^{\prime}(1)$ and $\pi^{-1}(1)>\left(\pi^{\prime}\right)^{-1}(1)$; or (b) $\pi(1)=\pi^{\prime}(1)$ or $\pi^{-1}(1)=\left(\pi^{\prime}\right)^{-1}(1)$ with some additional condition. The number of pairs $\left(\pi, \pi^{\prime}\right)$ satisfying each of the equations in (b) is $n(n-1) !^{2}$. (a) is equivalent to that (i) $2 \leq \pi(1)<\pi^{\prime}(1)$ and (ii) object 1 's rank in $\pi^{\prime}$ is higher than that in $\pi$. There are exactly $\left(\begin{array}{c}n-1 \\ 2\end{array}\right)$ pairs $\left\{\pi(1), \pi^{\prime}(1)\right\}$ of objects satisfying (i). The first ranks in the two orders being occupied by one such pair of objects, there are exactly $\left(\begin{array}{c}n-1 \\ 2\end{array}\right)$ ways to place object 1 subject to (ii). Finally, there are exactly $(n-2) !^{2}$ ways to fill the remaining ranks in the two orders. Thus, we have $N=\left(\begin{array}{c}n-1 \\ 2\end{array}\right)^{2}(n-2) !^{2}+O\left(n(n-1) !^{2}\right)$, and

$$
\mathcal{P}\left\{\pi>_{D} \pi^{\prime}, \pi^{\prime}>_{D}^{-} \pi\right\}=\frac{N}{n !^{2}}=\frac{\left(\begin{array}{c}
n-1 \\
2
\end{array}\right)^{2}(n-2) !^{2}+O\left(n(n-1) !^{2}\right)}{n !^{2}} \rightarrow \frac{1}{4}
$$

Part 2: $\succsim_{D}$ and $\succsim_{A}$ are asymptotically independent.

Proof. We count the number $N$ of pairs $\left(\pi, \pi^{\prime}\right)$ such that $\pi>_{D} \pi^{\prime}$ but $\pi^{\prime}>_{A} \pi$. This occurs if and only if either: (a) $\pi(1)<\pi^{\prime}(1)$ and $\pi(n)<\pi^{\prime}(n)$; or (b) $\pi(1)=\pi^{\prime}(1)$ or $\pi(n)=\pi^{\prime}(n)$ with some additional condition. The number of pairs $\left(\pi, \pi^{\prime}\right)$ satisfying each of the equations in (b) is again $n(n-1) !^{2}$. The number of combinations $\left\{\pi(1), \pi^{\prime}(1), \pi(n), \pi^{\prime}(n)\right\}$ satisfying (a) is $\left(\begin{array}{c}n \\ 2,2, n-4\end{array}\right)=\frac{n !}{2 ! 2 !(n-4) !}$. The number of pairs $\left(\pi, \pi^{\prime}\right)$ satisfying (a) is therefore $\left(\begin{array}{c}n \\ 2,2, n-4\end{array}\right)(n-2) !^{2}$. Hence $N=\left(\begin{array}{c}n \\ 2,2, n-4\end{array}\right)(n-2) !^{2}+O\left(n(n-1) !^{2}\right)$, and

$$
\mathcal{P}\left\{\pi>_{D} \pi^{\prime}, \pi^{\prime}>_{A} \pi\right\}=\frac{N}{n !^{2}}=\frac{\left(\begin{array}{c}
n \\
2,2, n-4
\end{array}\right)(n-2) !^{2}+O\left(n(n-1) !^{2}\right)}{n !^{2}} \rightarrow \frac{1}{4}
$$


Claim 2. Each lexicographic rule is asymptotically independent of $\gtrsim_{K}$.

Proof. In Steps 1-4 below, we will show that $\succsim_{D}$ and $\succsim_{K}$ are asymptotically independent. To deduce from this that $\succsim_{D}^{-}$and $\gtrsim_{I}$ are also asymptotically independent, recall the definition of $\gtrsim_{D}^{-}$as $\succsim_{D}$ applied to the inverses of permutations. It is easy to check that $I\left(\pi^{-1}\right)=I(\pi)$ for any $\pi$. Thus $\gtrsim_{D}^{-}$and $\succsim_{K}$ disagree as frequently as $\succsim_{D}$ and $\succsim_{K}$ do. It is therefore enough to establish the result for $\gtrsim_{D}$ and $\gtrsim_{K}$. The asymptotic independence between $\gtrsim_{A}$ and $\gtrsim_{K}$ and between $\gtrsim_{A}^{-}$and $\gtrsim_{K}$ can be similarly proved.

For each order $\pi$, define the left inversion vector $L(\pi)=\left(L_{i}(\pi)\right)_{i=1}^{n}$ and the right inversion vector $R(\pi)=\left(R_{i}(\pi)\right)_{i=1}^{n}$ by

$$
\begin{aligned}
& L_{i}(\pi)=\text { number of } j<i \text { such that }\{i, j\} \text { is an inversion in } \pi, \\
& R_{i}(\pi)=\text { number of } j>i \text { such that }\{i, j\} \text { is an inversion in } \pi .
\end{aligned}
$$

Obviously,

$$
I(\pi)=\sum_{i=1}^{n} R_{i}(\pi) .
$$

It is also well known that the mapping $R$ is a bijection from $\Pi$ to $\{0, \ldots, n-1\} \times\{0, \ldots, n-2\} \times \ldots \times\{0\}$. Define a new comparison rule $\succsim_{R}$ as follows:

- Rule $\gtrsim_{R}: \pi>_{R} \pi^{\prime}$ if and only if there exists $i \leq n$ such that $R_{j}(\pi)=R_{j}\left(\pi^{\prime}\right)$ for all $j<i$ and $R_{i}(\pi)<R_{i}\left(\pi^{\prime}\right)$, and $\pi \sim_{R} \pi^{\prime}$ if and only if $\pi=\pi^{\prime}$.

Define two random variables $X_{i}$ and $Y_{i}$ by

$$
X_{i}\left(\pi, \pi^{\prime}\right)=R_{n-i+1}(\pi), Y_{i}\left(\pi, \pi^{\prime}\right)=R_{n-i+1}\left(\pi^{\prime}\right) .
$$


Let

$$
D_{i}:=X_{i}-Y_{i}, S_{i}:=\sum_{j=1}^{i} D_{j}
$$

Step 1: $\gtrsim_{D}=\succsim_{R}$.

Proof. $\succsim_{D}$ is a linear order on $\Pi$. Recalling that $R$ is a bijection, it also follows that $\succsim_{R}$ is a linear order on $\Pi$. Thus it suffices to show that $\pi>_{D} \pi^{\prime}$ implies $\pi>_{R} \pi^{\prime}$. Suppose $\pi>_{D} \pi^{\prime}$. There exists $i$ such that: (a) $\pi(j)=\pi^{\prime}(j)$ for all $j<i$; and (b) $\pi(i)<\pi^{\prime}(i)$. (a) implies that $L_{j}(\pi)=L_{j}\left(\pi^{\prime}\right)$ for all $j<i$. But it is easy to see that

$$
\pi(j)=j-L_{j}(\pi)+R_{j}(\pi)
$$

for any $\pi$ and any $j$. Thus, $R_{j}(\pi)=R_{j}\left(\pi^{\prime}\right)$ for all $j<i$. (a) and (b) imply that $L_{i}(\pi) \leq L_{i}\left(\pi^{\prime}\right)$. Combining this inequality with (b), the above equation yields $R_{i}(\pi)<R_{i}\left(\pi^{\prime}\right)$. Therefore, $\pi>_{R}$ $\pi^{\prime}$.

Step 2: The random variables $D_{i}, 1 \leq i \leq n$, are independent with the following symmetric distributions:

$$
\mathcal{P}\left\{D_{i}= \pm x\right\}=(i-x) / i^{2}, 0 \leq x \leq i-1 \text {. }
$$

Moreover, $s_{i}:=\sqrt{\operatorname{Var}\left(S_{i}\right)}=i^{3 / 2} / 2 \sqrt{3}+o\left(i^{3 / 2}\right)$, and for each real number $x$,

$$
\mathcal{P}\left\{S_{n} / s_{n} \leq x\right\} \rightarrow \Phi(x)
$$

where $\Phi$ is the standard normal distribution function.

Proof. The proof of Step 2 is only technical and mostly coincides with a known proof that "the distribution of the number $I(\pi)$ of inversions in the randomly drawn permutation $\pi$, with some appropriate normalization, converges to the standard normal distribution" (see, e.g., Chung (2001)). Thus we defer it to the Appendix. 


\section{Step 3:}

$$
\mathcal{P}\left\{\pi>_{K} \pi^{\prime}, \pi^{\prime}>_{D} \pi\right\}=\sum_{x=1}^{n-1} \frac{n-x}{n^{2}} \mathcal{P}\left\{S_{n-1}<-x\right\}+O(1 / n) .
$$

Proof. By Step $1, \pi>_{K} \pi^{\prime}$ and $\pi^{\prime}>_{D} \pi$ occur at the same time if and only if either: (a) $D_{n}>0$ and $S_{n}<0$; or (b) $D_{n}=0$ with some additional condition. By Step 2, the probability that $D_{n}=0$ is $\frac{1}{n}$; hence the probability of (b) is $O(1 / n)$. Since $D_{i}$ 's are independent by Step 2, the probability of (a) can be written as $\sum_{x=1}^{n-1} \mathcal{P}\left\{D_{n}=x\right\} \mathcal{P}\left\{S_{n-1}<-x\right\}$, where, by Step $2, \mathcal{P}\left\{D_{n}=x\right\}=\frac{n-x}{x^{2}}$.

Step 4: $\mathcal{P}\left\{\pi>_{K} \pi^{\prime}, \pi^{\prime}>_{D} \pi\right\} \rightarrow \frac{1}{4}$.

Proof. Consider the right-hand side expression in Step 3. Observe that

$$
\sum_{x=1}^{n-1} \frac{n-x}{n^{2}} \mathcal{P}\left\{S_{n-1}<-x\right\} \leq \sum_{x=1}^{n-1} \frac{n-x}{n^{2}} \mathcal{P}\left\{S_{n-1}<-1\right\}=\frac{n-1}{2 n} \mathcal{P}\left\{S_{n-1}<-1\right\}
$$

Since $S_{n-1}$ is distributed symmetrically about $0, \mathcal{P}\left\{S_{n-1}<-1\right\} \leq \mathcal{P}\left\{S_{n-1}<0\right\}=1 / 2$. Hence $\lim \sup _{n \rightarrow \infty} \sum_{x=1}^{n-1} \frac{n-x}{n^{2}} \mathcal{P}\left\{S_{n-1}<-x\right\} \leq \frac{1}{4}$.

Now observe that

$$
\sum_{x=1}^{n-1} \frac{n-x}{n^{2}} \mathcal{P}\left\{S_{n-1}<-x\right\} \geq \frac{n-1}{2 n} \mathcal{P}\left\{S_{n-1}<-(n-1)\right\} .
$$

By Step 2, $\mathcal{P}\left\{S_{n}<-n\right\}=\mathcal{P}\left\{S_{n} / s_{n}<-n / s_{n}\right\} \sim \mathcal{P}\left\{S_{n} / s_{n}<-c / \sqrt{n}\right\}$ for a positive constant c. For any two numbers $n>N$ we have $\mathcal{P}\left\{S_{n} / s_{n}<-c / \sqrt{n}\right\} \geq \mathcal{P}\left\{S_{n} / s_{n}<-c / \sqrt{N}\right\}$. Letting $n \rightarrow \infty$, by Step 2 we have $\liminf _{n} \mathcal{P}\left\{S_{n} / s_{n}<-c / \sqrt{n}\right\} \geq \Phi(-c / \sqrt{N})$. Letting $N \rightarrow \infty$, we have $\liminf _{n} \mathcal{P}\left\{S_{n}<-n\right\} \geq 1 / 2$. Therefore, $\liminf _{n \rightarrow \infty} \sum_{x=1}^{n-1} \frac{n-x}{n^{2}} \mathcal{P}\left\{S_{n-1}<-x\right\} \geq \frac{1}{4}$. 


\section{Concluding remarks}

For small specific $n$, simple enumeration shows that the five comparison rules induce quite similar choice patterns. For $n=2$, there are only two orders $\Pi=\{12,21\}$ and each of the five rules places 12 over 21 . For $n=3$, each lexicographic rule always agrees with $\gtrsim_{K} \cdot{ }^{6}$ On the other hand, there are small positive chances that lexicographic rules disagree with each other. For example,

$$
\begin{aligned}
& 123>_{D} 132>_{D} 213>_{D} 231>_{D} 312>_{D} 321 \\
& 123>_{A} 213>_{A} 132>_{A} 312>_{A} 231>_{A} 321 \\
& 123>_{D}^{-} 132>_{D}^{-} 213>_{D}^{-} 312>_{D}^{-} 231>_{D}^{-} 321 \\
& 123>_{K} 132 \sim_{K} 213>_{K} 231 \sim_{K} 312>_{K} 321 .
\end{aligned}
$$

We observe that: $\succsim_{D}$ and $\succsim_{A}$ disagree at exactly two pairs of orders (i.e., with probability $\frac{2 \cdot 2}{3 !^{2}}=$ 0.11 ); $\gtrsim_{D}$ and $\gtrsim_{D}^{-}$disagree at exactly one pair of orders (i.e., with probability $\frac{2}{3 !^{2}} \sim 0.06$ ). For $n=4$, each lexicographic rule disagrees with $\succsim_{K}$ at some pairs of orders. For example, $1342>_{D} 2134$, but $2134>_{K}$ 1342. We can show that $\gtrsim_{D}$ and $\gtrsim_{K}$ disagree at exactly 17 pairs of orders (i.e., with probability $\frac{2 \cdot 17}{4 !^{2}} \sim 0.06$ ), and so on. These observations pose the question of whether some comparison rules have a positive amount of correlation bounded from zero for all $n$. In this paper we have shown that the answer is negative.

In some cases, our approach has limited applicability. For instance, in a social choice context where society selects an outcome based on reported preferences of individuals, Sato (2013a, b) explores implications of the assumption that "each individual makes only small lies." Sato assumes that each individual follows the Kemeny rule to rank preferences he may report, in order of "sincereness" (i.e., closeness to his true preference). It would be worthwhile to also consider the case where individuals adopt other comparison rules to assess the sincereness of their reported preferences. However, the result in this paper cannot be directly applied to demonstrate the impact of

\footnotetext{
${ }^{6}$ Here we say that two rules $\gtrsim$ and $\gtrsim^{\prime}$ agree on an object pair $\left\{\pi, \pi^{\prime}\right\}$ even if $\pi>\pi^{\prime}$ and $\pi \sim^{\prime} \pi^{\prime}$.
} 
alternative comparison rules in such circumstances, since the result itself tells us little about the extent to which the set of sufficiently sincere preference orders for an individual (i.e., his top $k$ preference orders for some small $k$ ) depends on his comparison rule. Future research may address this question.

Finally, we have focused on the choice situation where alternatives take the form of preference orders. Similar analysis may be conducted for other kinds of choice problems. One example is choice from subsets of objects. There is a large amount of literature on axiomatic characterizations of rules that extend an individual's preference over objects to his preference over subsets of objects (e.g., Bossert (1995)). ${ }^{7}$ It would be interesting to see whether a result like the one presented in this paper also holds for such extension rules satisfying natural axioms.

\section{Appendix: Proof of Step 2 for Claim 2}

The $2 n$ random variables $X_{i}$ and $Y_{i}, i=1, \ldots, n$, are independent with the following distributions:

$$
\mathcal{P}\left\{X_{i}=x\right\}=\mathcal{P}\left\{Y_{i}=x\right\}=1 / i, 0 \leq x \leq i-1 .
$$

It is well known that $X_{i}^{\prime}$ 's (or $Y_{i}^{\prime}$ 's) are independent and have the above distributions (Chung (2001) provides a very careful proof). Independence between $X_{i}$ 's and $Y_{i}$ 's is obvious. The distributions of $D_{i}$ 's described in Step 2 then follow from simple calculation. The following is also immediate:

$$
\begin{aligned}
& \operatorname{Var}\left(D_{i}\right)=\frac{i^{2}-1}{6} \\
& \operatorname{Var}\left(S_{i}\right)=\sum_{j=1}^{i} \frac{j^{2}-1}{6}=\frac{1}{36}\left(2 i^{3}+3 i^{2}-5 i\right) \\
& s_{i}=\sqrt{\operatorname{Var}\left(S_{i}\right)}=\frac{i^{3 / 2}}{2 \sqrt{3}}+o\left(i^{3 / 2}\right) .
\end{aligned}
$$

\footnotetext{
${ }^{7}$ One motivation that underlies works on such extension rules relates to individuals' incentives to manipulate the social choice correspondence; to describe such incentives, one needs to model each individual's ranking over subsets of outcomes.
} 
Now observe that the triangular array $\left\{\frac{D_{i}}{s_{n}} ; i=1, \ldots, n ; n=1,2, \ldots\right\}$ satisfies Lindeberg's condition. Thus, by the Lindeberg-Feller Central Limit Theorem, $S_{n} / s_{n}$ converges in law to the standard normal distribution.

\section{References}

Bossert, W. (1995) "Preference extension rules for ranking sets of alternatives with a fixed cardinality." Theory and Decision 39, 301-317.

Bossert, W., Sprumont, Y. (2014) “Strategy-proof preference aggregation: possibilities and characterizations." Games and Economic Behavior 85, 109-126.

Bossert, W., Storcken, T. (1992) "Strategy-proofness of social welfare functions: the use of Kemeny distance between preference orderings." Social Choice and Welfare 9, 345-360.

Burak, C., Storcken, T. (2013) "A re-characterization of the Kemeny distance." Maastricht GSBE Research Memoranda, RM/13/009.

Chung, K.L. (2001) A Course in Probability Theory, 3rd ed. Academic Press, San Diego, CA.

Kemeny, J.G., Snell, J.L. (1962) Mathematical Models in Social Sciences. Blaisdell, New York.

Laffond, G., Lainé, J.(2000) "Majority voting on orders.” Theory and Decision 49, 251-289.

Rubinstein, A., Salant, Y. (2012) "Eliciting welfare preferences from behavioural data sets." Review of Economic Studies 79, 375-387.

Sato, S. (2013a) "A sufficient condition for the equivalence of strategy-proofness and nonmanipulability by preferences adjacent to the sincere one." Journal of Economic Theory 148, 259-278. 
Sato, S. (2013b) "Strategy-proofness and the reluctance to make large lies: the case of weak orders." Social Choice and Welfare 40, 479-494.

Sato, S. (2013c) "Bounded response and the equivalence of nonmanipulability and independence of irrelevant alternatives." mimeo. 